\title{
RAE
}

\section{Patrones y técnicas}

\section{Biomecánica y análisis del desarrollo motor}

\author{
DANIEL IGNACIO OLIVEROS WILCHES* \\ JHON PETER MUNOZ**
}

\section{Resumen}

ste proyecto se centra en el estudio de los patrones de movimiento y en las técnicas de movimiento que se incluyen en la cultura de la educación física colombiana, en particular en la de Bogotá. Intenta describir cómo se están desarrollando los patrones de movimiento fundamentales en niños y niñas de 6 a 12 años, en algunos colegios de Bogotá, observados y evaluados desde una perspectiva cualitativa, basada en principios de biomecánica.

Palabras clave: desarrollo motor, patrones motores, biomecánica, análisis del movimiento, evaluación en educación física.

\section{Objetivos}

El propósito general de esta investigación es conocer sobre la estructura de los patrones y técnicas de movimiento que la cultura colombiana promueve en las actividades de las clases de educación física en los diferentes entrenamientos deportivos y en las actividades físicas.

También, en forma específica se pretende:

- Determinar qué patrones motores fundamentales se están promoviendo en las clases de educación física en niños y niñas de 6 a 12 años.

- Precisar en qué nivel de desarrollo se encuentran tales patrones.
- Desarrollar un modelo de evaluación de los patrones de movimiento desde una perspectiva biomecánica.

\section{Referentes teóricos}

Diversos autores (Seefeldt, 1966; Wickstrom, 1983; MaClenaghan y Gallaghue, 1986) han establecido que el desarrollo motor de los primeros años no es un producto exclusivo de la maduración biológica, sino que las experiencias de movimiento son importantes para su desarrollo, y necesarias para el máximo perfeccionamiento y desarrollo de los patrones de movimiento del adulto.

\footnotetext{
* Investigador principal. Profesor Faculatd de Educación Física. Universidad Pedagógica Nacional.

** Coinvestigador. Profesor Catedrático. Universidad Pedagógica Nacional.
} 
El concepto de estructura de movimiento propuesto por Meinel (1971) y sistematizado por Karl Koch (1981: 162-163) representa un medio posible para objetivar el movimiento, que es fugaz, único e irrepetible y posible de ser interpretado significativamente desde el sujeto que lo ejecuta o quien lo observa o quien recibe su influencia física. El análisis dinámico (fuerzas y conceptos relativos) revela la estructura dinámico motriz. Esta visión no alude a la estructura de significado que se pueda dar desde otras perspectivas de estudio. Meinel también propuso un análisis de los movimientos fundamentales, en la perspectiva del desarrollo motor.

Knudson y Morrison (1997), al proponer un enfoque cualitativo de análisis del movimiento, plantean dos categorías de modelos: los modelos comprensivos que intentan resumir todas las tareas importantes que son relevantes para un análisis del movimiento humano; y los modelos que se concentran en la tarea de la observación del movimiento, que a su vez pueden ser pedagógicos y biomecánicos, dependiendo del foco de análisis.

Los estudios científicos sobre el tema (Seefeldt, 1966; Wickstrom,1983; MaClenaghan y Gallaghue, 1986; Meinel, 1971; Karl Koch; Cratty 1982; Bequer 1996; Muñoz, 1991), presentan perspectivas, conclusiones y recomendaciones sobre el desarrollo motor. Las perspectivas son amplias, pero se quiere sintetizar una de carácter biomecánico que permita un enfoque de aplicación cualitativo para la enseñanza y valoración del desarrollo motor. Las ideas de estos autores, en algunos casos, no se han llevado a la práctica en la labor de los profesores de educación física. Un enfoque cualitativo, basado en una perspectiva biomecánica, permitiría sintetizar varias de las visiones en un modelo aplicable a la realidad profesional.

Los estudios mencionados sólo se han ocupado de niños de cero a seis años, con excepción de los de Wickstrom y Cratty, y la propuesta de Knudson y Morrisson, no menciona la población. De otro lado, los niños estudiados pertenecen a poblacio- nes de distintas culturas a la colombiana, con excepción del trabajo de Muñoz.

Se aprecia en la revisión que no hay un acuerdo sobre cuáles son los patrones básicos o fundamentales. Es decir, no hay una universalización del concepto, que se acomode a las diferentes culturas. Tampoco se mencionan niveles del proceso como se deben enseñar o adquirir tales patrones. Se consideran un paquete, pero no hay prioridades o jerarquías para ser enseñados o asimilados por los niños y jóvenes. Son muy escasas las referencias cronológicas sobre aparición y posibilidades de intervención para la maduración de los patrones.

En relación con la intervención de los maestros o entrenadores sobre los patrones y combinaciones, no se encuentran propuestas sobre el tiempo que se emplea en adquirir y madurar un patrón, en relación con la cronología del desarrollo. Es decir, un profesor no dispone de referencias para saber en qué edad puede intervenir sobre un patrón, ni tampoco se sabe durante cuánto tiempo debe hacerlo para lograr una maduración elevada.

Algunos autores han considerado distintos patrones motores fundamentales o básicos. Wickstrom (1983) propuso los siguientes: andar, correr, saltar, lanzar, coger (tomar con la mano), golpear y dar patada (patear). Lucea (1999), propone los siguientes: caminar, correr, saltar, coger, lanzar, golpear, rodar, reptar y trepar; los denomina esquemas motores, pero reconoce el nombre de patrones motores. Para Vern Seefeldt las destrezas motoras fundamentales son: caminar, correr, agarrar, jalar, lanzar, empujar, golpear, gatear, deslizar, caer, galopar, saltar, patear, rodar, patinar y girar.

Los profesores de educación física deberían disponer de conocimiento biomecánico e integrarlo en su formación y trabajo profesional. Los movimientos fundamentales pueden ser analizados desde la perspectiva del cuerpo total y de las partes que se mueven, de los resultados del movimiento o desde las condiciones que rodean un aprendizaje; 
pero también desde los principios biomecánicos que se manifiestan o se violan en la realización de movimientos.

\section{Metodología}

Este estudio se centra en niños y niñas de seis a doce años de colegios de básica primaria. Es un estudio sincrónico.

La investigación que se propone está basada en:

- Construcción de unos modelos teóricos de patrones motores fundamentales desde la perspectiva biomecánica (preparación del modelo).

- Análisis y valoración de movimientos: por medio de filmaciones y observaciones sistematizadas de movimientos de poblaciones seleccionadas (observación sistematizada).

- Determinación de los niveles de desarrollo encontrado en las poblaciones observadas (evaluación/diagnóstico).

- Con base en los resultados de estas observaciones se construiría una propuesta de relaciones entre patrones y sus combinaciones, como estructura sugerida para la orientación del desarrollo motor de los niños y jóvenes.

La biomecánica es una ciencia empírica analítica y sus métodos de investigación presentan características correspondientes. Sin embargo, en este estudio se usan los conocimientos sistemáticamente obtenidos de ésta disciplina y se aplican en el dịseño de un modelo para evaluar/diagnosticar el desarrollo motor de niños. Otras perspectivas metodológicas han enfatizado la medición de los resultados del movimiento, usando tests estandarizados, construyendo tablas de comparación y mostrando cómo evolucionan las marcas con la edad y cómo se diferencian los resultados en los dos géneros (Malina, por ejemplo). Sin embargo, la metodología que se propone permite la intervención del maestro, favoreciendo que el proyecto se desarrolle como práctica pedagógica de los estudiantes de la Facultad y que se aprecien los cambios en la estructura de movimiento en relación con el tiempo. Es entonces, un trabajo descriptivo.

\section{Resultados esperados}

Los resultados que se esperan son la proposición de:

- Un modelo de patrones motores fundamentales, con descripción, fases, imágenes, factores críticos. Se producirán en medio físico y electromagnético.

- Una metodología de análisis cualitativo y cuantitativo de patrones de movimiento.

- Una descripción sobre el nivel de desarrollo de los patrones en escolares.

- El establecimiento de relaciones de secuencia u orden en el desarrollo de los patrones y sus combinaciones.

\section{Bibliografía}

BEQUER, Gladys. (1996). Desarrollo motor: Clasificación de los estadios, tratamiento metodológico. Mímeo del posgrado en pedagogía de la educación física. La Habana.

BURTON, Allen y MLLER, Daryl. (1998). Movement Skill Assesment. Human Kinetics: Champaign, Ill.

CRATTY, Bryant. (1990). Desarrollo perceptivo y motor en los niños. Paidós.

GODFREY, B. y KEPHART, N. C. (1969). Movementspatterns and motor education. Nueva York. Appleton - Century - Croffs
KNUDSON, Duane y MORRISON, Craig. (1997). Qualitative Analysis of Human Movement. Human Kinetics: Champaign, Ill.

KOCH, Karl. (1973). Carrera, salto y lanzamiento en la escuela elemental. Buenos Aires. Kapelusz.

Aires. Kapelusz.

Hacia una ciencia del deporte. (1981). Buenos

LAROVERE, Paul. Movimientos Básicos. www.sobreentrenamiento.com. 
MALINA, Robert y BOUCHARD, Claude. (1991). Growth, Maturation, and Pbysical activity. Human Kinetics: Champaign, III.

MCCLENAGHAN, Bruce y GALLAGHUE, David. (1985). Movimientos fundamentales: una aproximación de desarrollo y remedial. Buenos Aires. Medica Panamerina.

MFINEI, Kurt. (1977). Didáctica del Movimiento. Habana. Orbe.

MUÑOZ, Luis Armando. (1991). Evaluación de habilidades motoras y crecimiento de niños en un jardín. En Revista de Educación Física y Deportes. Medellín, Colombia. Vol. 13, № 1-2. (1994). Educación psicomotriz: Manizales. Kinesis.
RUIZ, Pérez y M. Luis. Desarrollo motor y actividades fisicas. Madrid. Gymnos.

SEEFELDT, Vern. (1996). Developmental motor patterns: Implications for elementary school physical education. En: Memorias del Congreso de Educación Física y Actividad Física. Quebec, Canada.

WICKSTORM, R. (1983). Patrones motrices básicos. Madrid. Alianza Deporte. 